\title{
Desequilibrios externos globales y cooperación internacional: ¿Dónde estamos?
}

\author{
JUAN TUGORES QUES \\ Universidad de Barcelona, Fctad. de Economía y Empresa, Avda. Diagonal, 696, 08034 Barcelona, \\ España.E-mail: jtugores@ub.es
}

\begin{abstract}
RESUMEN
Las preocupaciones por los grandes desequilibrios externos en los años previos a la crisis parecen haberse reducido a medida que su magnitud lo ha hecho. Pero hay dudas acerca de si las fragilidades y distorsiones a que respondían se han visto corregidas en sus causas de fondo o si, por el contrario, subsisten. La cooperación internacional en el marco del G20 trata explícitamente de afrontar esos desequilibrios pero las asimetrías en su corrección pueden estar teniendo efectos recesivos. Unos rendimientos decrecientes a la cooperación internacional tratan de ser revertidos con planteamientos que propicien una mejora canalización del ahorro hacia la inversión productiva en unos marcos institucionales nacionales y globales de mayor calidad, pero subsisten muchas dificultades
\end{abstract}

Palabras clave: Desequilibrios externos, cooperación internacional, coordinación internacional, crisis financiera

\section{Global External Imbalances and International Cooperation: Where do we stand?}

\begin{abstract}
Concerns about large external global imbalances in the pre-crisis years seem to be weakening as their magnitude decreases. But doubts remain about whether the root causes of the weaknesses and distortions which triggered these imbalances have in fact been corrected. International cooperation in the framework of the G20 aims explicitly at tackling these imbalances, but an asymmetric correction may be having recessionary effects. New approaches for a more ambitious strategy are trying to revert diminishing returns to international cooperation to promote growth and a better channeling of savings into productive investment in an improved institutional framework both at a national and a global level. However, many difficulties remain.
\end{abstract}

Keywords: External Imbalances, International Cooperation, International Coordination, Financial Crisis.

Clasificación JEL: F42, F41, F32

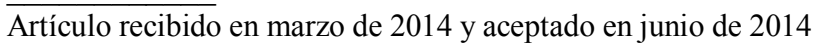

Artículo disponible en versión electrónica en la página www.revista-eea.net, ref. ə-32308 


\section{INTRODUCCIÓN}

A mediados de la primera década del siglo XXI el debate sobre los desequilibrios externos se aupaba al primer nivel de las preocupaciones en la economía financiera internacional. Desde 2005 la atalaya del World Economic Outlook del FMI se hacía eco de las preocupaciones al respecto tras una sección del mismo informe ya en la edición de septiembre de 2002. El déficit por cuenta corriente de Estados Unidos se contraponía al superávit de China, pero asimismo otras economías avanzadas -incluida la "periferia Sur" de la eurozona- entraban en una trayectoria de déficits contrapuesta a unos países con importantes superávits entre los que se encontraban Alemania y Japón, pero en que la mayor parte de los principales superavitarios eran países exportadores de petróleo y otras materias primas o las nuevas potenciales comerciales asiáticas. A las dimensiones más estrictamente económico-financieras se le superponían consideraciones geoestratégicas. A los debates más "técnicos" acerca de qué depreciación del dólar sería necesaria para reconducir el déficit de Estados Unidos a niveles sostenibles se les añadía la preocupación por las fragilidades de los que los desequilibrios podrían ser síntomas y que podían conducir a una crisis ${ }^{1}$.

Cuando estalla la crisis se amplía el debate al papel de los desequilibrios externos en su gestación. En ocasiones se trata de contraponer la "responsabilidad" de tales desequilibrios a la que habrían tenido otros factores causales como los excesos en el binomio de la excesiva expansión crediticia y su contrapartida del excesivo endeudamiento de muchos agentes económicos aunque se reconocen las interconexiones entre ambas fuentes, especialmente por la vía de cómo la disponibilidad de financiación exterior abundante y barata incluso en países con ahorro doméstico insuficiente habría propiciado la asunción de riesgos y una asignación de los recursos hacia ámbitos de productividad subóptima. La propia dinámica de la crisis habría reducido inicialmente la magnitud de los desequilibrios, en parte por un repliegue inicial de los flujos comerciales y financieros internacionales, en parte por la orientación más doméstica en la asignación de recursos por parte de algunos países superavitarios, aunque quedaba abierta la controversia acerca de si la recuperación económica volvería a venir acompañada de un nueva ampliación de los desequilibrios. Los desequilibrios externos se incorporan desde el inicio a la agenda de la cooperación internacional en el marco del G20. Desde la cumbre de Pittsburgh (2009) se asume un Framework for Strong, Sustainable and Balanced Growth con recomendaciones explícitas tanto a los países con superávits y déficits "sostenidos y significati-

\footnotetext{
${ }^{1}$ Los artículos del volumen 2005.1 de Brookings Papers on Economic Activity son referencias ya clásicas, así como los de Economic Policy de julio de 2007 (vol. 22, issue 51). Pero pronto se evidenció que solucionar los desequilibrios iba mucho más allá de reajustes cambiarios $\mathrm{y}$, además, al inicio de la crisis el papel del dólar como moneda-refugio prevaleció sobre las eventuales desconfianzas asociadas al gran déficit exterior de Estados Unidos.
} 
vos" en cuyo seguimiento, a través de un Mutual Assessment Process (MAP) el FMI tiene un papel destacado.

En Europa, a las controversias sobre los desequilibrios externos globales se les superponía de forma creciente la preocupación por los "desequilibrios externos internos a la eurozona", a la vista de cómo en los primeros años del siglo XXI se ampliaban de forma muy notable las asimetrías entre las posiciones superavitarias de algunos países de la zona euro, con Alemania al frente, y los déficits por cuenta corriente de otros países especialmente en la periferia Sur. Las "interpretaciones benignas" fueron dando paso a preocupaciones acerca de las tensiones que ello originaba para el proceso de integración monetaria, incluida su propia sostenibilidad. Desde 2012 la UE tiene operativo el Macroeconomic Imbalance Procedure (MIP) para tratar de prevenir y corregir los problemas reconocidamente graves, siendo la evolución del saldo por cuenta corriente el primero de los 5 indicadores de la categoría "desequilibrios externos y competitividad" 2 .

Como consecuencia de las dinámicas y ajustes postcrisis se producían algunos cambios en la composición de los países más afectados y en las estimaciones y valoraciones de la magnitud de los desequilibrios. Mientras del ranking ${ }^{3}$ de países que encabezaban las "importaciones de capital" desaparecían algunos del Sur de Europa y aparecían algunos destacados emergentes como Brasil e India, por el lado de los "exportadores de capital" Alemania reemplazaba a China en la cabeza del listado. Respecto a la cuantía de los desequilibrios y su previsible evolución futura, la forma con que los documentos de organismos internacionales como el FMI y la OCDE se referían al tema eran exquisitamente cautelosos, haciéndose eco del papel al respecto de la "compresión de la demanda" en las economías avanzadas, al tiempo que el FMI insistía (y la declaración final del G20 en San Petersburgo se hacía eco ${ }^{4}$ ) en que "las políticas han desempeñado un papel relativamente menor" en la reducción de los desequilibrios y hacían falta más esfuerzos al respecto, entre otras cosas para "evitar un resurgimiento de los desequilibrios globales a medida que avance la recuperación”.5. De forma más optimista, a principios de 2014 Barry Eichengreen daba

\footnotetext{
${ }^{2} \mathrm{El}$ tema de los desequilibrios por cuenta corriente intraeuropeos merece tratamiento específico y desborda el ámbito de este artículo, por lo que nos referiremos a ellos únicamente en los aspectos que sean una dimensión especialmente relevante de los desequilibrios a escala global.

${ }^{3}$ Según los datos extraídos de los apéndices del Global Financial Stability Report del FMI (varias ediciones)

${ }^{4}$ El texto coincidente del Informe del FMI a la cumbre del G20 y el texto de la Declaración de los Líderes de San Petersburgo -FMI (2013b) y G20 (2013)- es explícito al respecto. En paralelo, el FMI y la OCDE publican documentos con "descomposiciones" de la variación de los desequilibrios entre ingredientes cíclicos y estructurales, como se comenta más adelante.

${ }^{5}$ FMI (2014).
} 
por finiquitado el problema de los desequilibrios globales con algunos matices importantes el principal de los cuales se refería precisamente al superávit de Alemania $^{6}$.

¿Dónde estamos? ¿Han abandonado los desequilibrios globales la larga lista de preocupaciones graves o es prematuro darlos por finiquitados?. Este artículo revisa y sistematiza algunos de los importantes temas que subyacen a estas controversias. Su hilo conductor es que los desequilibrios externos han sido en parte un síntoma acerca de una forma de funcionar el sistema económico y financiero -tanto a escala global como europea e interna de muchos países- pero a partir de ciertos umbrales se han convertido también en causas de fragilidades y problemas. Por ello es importante valorar en qué medida se han corregido los síntomas, pero sobre todo los problemas de fondo, entre ellos algunos tan cruciales como equilibrio ahorro-inversión, pautas razonables de competitividad más sólida para fundamentar sin nuevas fragilidades la recuperación o la calidad institucional para propiciar esos ajustes y supervisarlos a escala nacional y supranacional. ¿Se ha avanzado más en la corrección de los síntomas que en la de los problemas de fondo? O, incluso más preocupante, la reducción en la magnitud estadística de los desequilibrios externos $¿$ se ha realizado de una forma inadecuada, en la que ha habido excesivas asimetrías en los ajustes y se han generado impactos recesivos ${ }^{7}$ que pueden estar contribuyendo a la lentitud y fragilidad de la recuperación?. Y en la medida que, al menos parcialmente, pueda considerarse que ha sido así, qué perspectivas hay de correcciones, y qué papel pueden desempeñar los mecanismos de cooperación internacional? ¿Se encuentra esta coordinación internacional sujeta a "rendimientos decrecientes", como a veces se apunta, o iniciativas como la agenda anunciada para Brisbane 2014 puede estar suponiendo un intento serio de revitalizar la coordinación internacional y su efectividad?.

Para ofrecer algunas respuestas a estas cuestiones la sección 2 resume algunos de los principales conceptos y datos a tener en cuenta. La sección 3 analiza los problemas que surgen en el crucial mecanismo de la captación del ahorro y su canalización eficiente a través del sistema financiero -nacional y globalhacia la inversión productiva...o no. La sección 4 valora los intentos de efectuar un seguimiento de los desequilibrios globales, articular mecanismos de cooperación y las dificultades para conseguirlo. La sección 5 ofrece unas consideraciones finales.

\footnotetext{
6 "Un réquiem por los desequilibrios globales" es el explícito título de su artículo.

7 "Desequilibrios internacionales con ajustes asimétricos y con sesgo recesivo" es el explícito título de un epígrafe del capítulo I del Trade and Development Report 2013 de UNCTAD (2013).
} 


\section{ALGUNOS DATOS Y CONCEPTOS POLIÉDRICOS}

Clarificar el alcance y sentido de los desequilibrios externos globales requiere explicitar varias de sus poliédricas vertientes, cada una de las cuales ofrece perspectivas importantes que se complementan.

\subsection{Saldos por cuenta corriente y la cuenta financiera}

Pese a ser aparentemente casi obvio, debe recordarse que los desequilibrios pueden enfocarse desde la perspectiva de las balanzas por cuenta corriente (y en su caso, la balanza por cuenta de capital) de los países implicados, o bien desde la perspectiva de la cuenta financiera. Este segundo enfoque concede un papel central a las discrepancias entre ahorro e inversión domésticos, mostrando unos países capacidad de financiación al resto del mundo mientras que en otros casos la insuficiencia del ahorro interno para financiar la inversión conduce a necesidad de financiación exterior. Estos saldos netos entre ahorro e inversión son la resultante de una multiplicidad de transacciones con implicaciones jurídicas, empresariales y financieras heterogéneas. Desde la perspectiva de la cuenta corriente, unos países se encuentran con un déficit, que deben financiar, por una situación en que la absorción supera a la producción, con eventuales problemas de competitividad. Y otros países se encuentran en situación de superávit por cuenta corriente, resultado de una absorción por debajo de su producción interna $\mathrm{y}$, en su caso, un buen posicionamiento competitivo. Los análisis tradicionales sobre políticas de variación de gasto y/o el papel de los tipos de cambio enlazan con este segundo enfoque. Las identidades de la balanza de pagos deberían asegurar la coherencia entre ambos enfoques, pero la magnitud de las "discrepancias estadísticas" y los "errores u omisiones", por un lado, y el papel especial de ajuste de las variaciones de reservas internacionales por otro, convierten a ambos enfoques en más complementarios que reiterativos ${ }^{8}$.

\subsection{Unos datos básicos}

Es bien conocida la dinámica al alza de los desequilibrios desde finales del siglo XX. En 2007 el superávit por cuenta corriente de China era del 10,1\% de su PIB, mientras que el déficit de Estados Unidos se situaba en el 4,9\% del suyo (tras haber alcanzado el 2006 el 5,8\%), Los datos expresados en porcentajes del

\footnotetext{
${ }^{8}$ Otra dimensión destacada es cómo las variables-stock relativas a Posiciones Financieras Netas de los países no coinciden en su dinámica con la evolución de los (flujos de los) saldos por cuenta corriente en la medida que haya alteraciones importantes en las valoraciones de activos y pasivos, además de variaciones en los tipos de cambio. Gourinchas y Rey (2013) examinan con detalle el importante papel de estos efectos valoración.
} 
PIB mundial el déficit USA llegó a situarse en el 1,5\%. En términos absolutos ${ }^{9}$, los desequilibrios globales -medidos por la suma del valor absoluto de los principales déficits y de los principales superávits- alcanzaban el 5\% del PIB mundial a principios de 2008 pero a finales de 2012 la magnitud de los desequilibrios se había reducido sustancialmente, hasta el 2,5\% del PIB mundial. El déficit de Estados Unidos se ubicaba en el 2,7\% de su PIB, mientras que el superávit de China se había reducido al 2,3\%. Las economías del Sur de Europa habían pasado de fuertes posiciones deficitarias a prácticamente equilibrio, siendo el elemento discordante en esta dinámica el caso de Alemania cuyo superávit alcanzaba el $7 \%$ de su $\mathrm{PIB}^{10}$, activando incluso la señal de alarma prevista al respecto en el MIP.

Un tema de controversia es el papel de los factores coyunturales/cíclicos en esa reducción reciente de los desequilibrios. Análisis desde la OECD concluyen que, en promedio, en torno a la mitad se puede atribuir a motivos coyunturales asociados a la contracción/ralentización de la actividad ${ }^{11}$. Este debate plantea dos cuestiones: por un lado, los factores estructurales que van más allá de las fluctuaciones estrictamente cíclicas; por otro, los riesgos de que la recuperación vaya acompañada por un resurgimiento de los desequilibrios.

\subsection{Desequilibrios apropiados o inapropiados}

En las "radiografías" de los datos hay dimensiones más detalladas y que afectan tanto a aspectos cuantitativos como valorativos. Que las cuentas exteriores no estén equilibradas no significa necesariamente unos desequilibrios en el sentido peyorativo del término. Como en la vida económica de las familias y empresas, hay situaciones y momentos en que una posición deficitaria está justificada como resultante de decisiones racionales: apelar a financiación exterior para poder complementar un limitado ahorro interno y así poder movilizar nuevas excelentes oportunidades de inversión es el ejemplo clásico. Y ejemplos análogos pueden referirse a la justificación de posiciones superavitarias. Pero asimismo pueden encontrarse situaciones en que los desequilibrios o bien han ido más lejos de lo racionalmente justificable o bien derivan de decisiones inadecuadas resultantes de distorsiones o ineficiencias. Blanchard y Milesi-Ferretti (2009) es una excelente descripción de cómo fueron fluctuando las expli-

\footnotetext{
${ }^{9}$ Técnicamente, la suma de superávits debería igualar a la de déficits, pero incluso las gráficas en que típicamente el FMI presenta la evolución de los global imbalances suele añadir la línea de "discrepancias" que en varios momentos ha superado el $0,5 \%$ del PIB mundial.

${ }^{10}$ Datos del apéndice estadístico del World Economic Outlook del FMI, octubre 2013.

${ }^{11}$ Ollivaud y Schwellnuss (2013) desde OECD atribuyen la mitad de la reducción a factores del ciclo económico y de la vivienda, y una quinta parte a los ajustes fiscales y cambiarios. Asimismo FMI (2013c). En el caso de los países del Sur de Europa, un análisis del ECB (2014) apunta a que menos de la mitad de la corrección se habría debido a factores cíclicos.
} 
caciones y valoraciones en el camino hacia la ampliación de los desequilibrios en los años inmediata anteriores a la Gran Recesión, pasándose de una etapa en que las interpretaciones "benignas" podían estar justificadas a otra en que era cada vez más difícil apelar a nociones de "optimización intertemporal" o similares para eludir la conclusión de importantes fragilidades y riesgos.

Una forma de trasladar a la terminología las diferentes valoraciones de los datos en que se plasman los desequilibrios externos es la que plantea el 2013 IMF Pilot External Sector Report: utiliza la expresión pretendidamente "aséptica" current account divergences para describir las posiciones superavitarias/deficitarias de los diferentes países resultante de las estadísticas, reservado la referencia a external imbalances para la brecha entre las posiciones efectivas por cuenta corriente y las que se estiman consistentes con los fundamentals y las políticas "deseables". Con este enfoque la valoración de la reducción de los desequilibrios es menos optimista, apuntándose a la necesidad de estrategias adicionales para "cerrar los desequilibrios globales"

\subsection{Asimetrías entre déficits y superávits}

Un tema recurrente al tratar los desequilibrios son las asimetrías entre posiciones superavitarias y deficitarias. Los argumentos básicos acerca de los problemas de déficits exteriores excesivos se vinculan a deficiencias de competitividad, insuficiencias de ahorro doméstico y problemas de sostenibilidad que requieren solución al menos a medio plazo. Y cuando los acreedores dejan de proveer financiación o la encarecen la presión para corregir los déficits es notoria. En cambio los problemas asociados a posiciones superavitarias -y los incentivos a corregirlas- parecerían menos inmediatos. Entre ellos se incluyen argumentaciones relativas a cómo los superávits "excesivos" se vinculan a distorsiones internas, como insuficiente inversión o exceso de ahorro ${ }^{13}$-justificando así una coincidencia entre objetivos domésticos de reducir esas distorsiones y la contribución al objetivo global de reducir los desequilibrios también en los países superavitarios- , así como argumentos acerca de dimensiones globales, desde referencias a un "neomercantilismo" -y asociado a él, una peligrosa concepción de la economía mundial como un juego de suma ceroy los conflictos asociados a la críticas de que los superávits externos se logran, en alguna medida, mediante mecanismos de "políticas de empobrecer al vecino", un terreno resbaladizo pero que, como han resumido Blanchard y MilesiFerretti (2011) se enfrenta al dilema de que "demostrarlo es difícil, ignorarlo puede ser injusto".

\footnotetext{
${ }^{12}$ Vid. FMI (2013 a) para los detalles.

${ }^{13}$ En el caso de Europa, son los argumentos básicos que se pueden encontrar en Comisión Europa $(2012,2014)$ siendo especialmente debatidos en relación a Alemania.
} 


\subsection{Nuevas realidades, nuevas estadísticas}

La profundidad de los cambios que suponen las nuevas realidades globales ha conducido a revisar algunos criterios en la elaboración de las estadísticas con impactos en algunas mediciones (y subsiguientes valoraciones) de los desequilibrios globales.

Por un lado, el creciente papel de las redes globales de producción -global value chains- en que muchos de los componentes o de las actividades (tareas) que se incorporan a un producto final se originan en una multiplicidad de localizaciones a escala global, ha conducido a complementar las estadísticas tradicionales -en que a cada país se le imputa el valor bruto de los productos que exporta- por los datos en "valor añadido" en que a cada país se le imputa únicamente ese valor incorporado en su territorio. Los resultados modulan significativamente la "radiografía" de los flujos comerciales bilaterales. Y en particular, la dimensión más seguida de estos desequilibrios, la relación entre China y Estados Unidos, ve revisada a la baja su magnitud en aproximadamente una tercera parte, con los datos referidos $2008^{14}$. Ello debería contribuir a reducir las tentaciones de utilizar esos desequilibrios como "coartada" para tratar de promover posicionamientos más agresivos y/o proteccionistas.

Por otra parte, el enfoque del dark matter ${ }^{15}$ apunta a que, en su versión más radical, a que en buena medida, los superávits/déficits exteriores medidos por las estadísticas tradicionales podrían ser un "gigantesco error de contabilidad" cuya adecuada corrección llevaría a minimizar la magnitud de los desequilibrios. Los aspectos más relevantes de discrepancia hacen referencia al papel de activos inmateriales e intangibles que no estarían adecuadamente incorporados en las estadísticas tradicionales y que infravalorarían el know-how y otros aspectos, así como el papel desempeñado por el sistema financiero estadounidense como "banquero global" al reciclar los excedentes de países superavitarios con un grado de fluidez, solvencia y seguridad que los sistemas financieros de esos países no estaban en condiciones de ofrecer. Más allá de los aspectos más mediáticos de la controversia, constatar cómo son mejorables las formas de valorar activos intangibles, activos inmateriales, confianza (incluida fiabilidad asociada a la calidad institucional). Más adelante volveremos sobre algunas implicaciones, entre ellas la demanda y provisión de activos de la máxima seguridad ${ }^{16} \mathrm{y}$

14 Con los datos obtenidos a partir de la base de datos OECD-WTO disponible en: http://www.oecd.org/industry/ind/measuringtradeinvalue-addedanoecd-wtojointinitiative.htm

${ }^{15}$ Hausmann y Sturzenegger (2007)

${ }^{16}$ Ricardo Caballero (2010) ha insistido en el "otro desequilibrio": la escasez de activos realmente seguros, de máxima solvencia, en comparación con la demanda creciente que suscitó la globalización financiera. Cuestiones asociadas a ella es el papel de Estados Unidos como emisor de esos activos de máxima calidad, los excesos cometidos al generar una "oferta artificial de acti- 
los debates sobre el insuficiente grado de desarrollo financiero en las economías emergentes, sus fragilidades y retos.

\section{AHORRO, INVERSIÓN Y TIPOS DE INTERÉS A ESCALA GLOBAL}

Pocos meses antes de su nombramiento como presidente de la Reserva Federal, Ben Bernanke (2005) analizó las nuevas realidades en el mecanismo de canalización del ahorro hacia la inversión a escala global en su formulación del global savings glut. Esta denominación quería explicitar cómo los países en que el ahorro doméstico se situaba significativamente por debajo de la inversión doméstica -entre ellos Estados Unidos y algunos europeos- podían obtener acceso a financiación exterior a tipos de interés bajos gracias a que en el conjunto de la economía mundial había otros lugares con abundante ahorro que, además, estaban dispuestos a prestar a los países con "necesidad de financiación". Merece especial atención el énfasis de Bernanke en dos aspectos: a) la constatación de que incluso para Estados Unidos era muy relevante en una economía financieramente globalizada la correlación entre ahorro e inversión a escala global y no sólo nacional ; b) cómo la transición a posiciones excedentarias en algunas economías asiática se habría visto propiciado por las duras lecciones aprendidas en "su" crisis en 1997-1998, cuando constataron las fragilidades derivadas de una dependencia excesiva de un ahorro exterior que podrían haber llegado a creer, ingenuamente, que sería indefinidamente abundante y barato. El grado en que las economías occidentales más negativamente afectadas por la crisis estarían realizando (o no) un análogo proceso de aprendizaje merece atención así como los riesgos de que algunas economías emergentes hayan incurrido de nuevo en fragilidades en los últimos años ante una llegada importante de flujos financieros que, con la anunciada reversión de la expansión cuantitativa en Estados Unidos, podrían ralentizarse, de forma abrupta en algunos casos.

\subsection{Tipos de interés bajos...¿hasta ahora?}

En el planteamiento de Bernanke, el test crítico de que a escala global el ahorro era a principios del siglo XXI relativamente abundante era el bajo nivel de los tipos de interés. Incluso considerado artificialmente bajo, según diversos criterios $^{17}$, en países como Estados Unidos o el Sur de Europa, lo que habría

vos sintéticos" presentados como de máxima calidad pero con fundamentos débiles, y el debate sobre la provisión de esos activos de máximo nivel en un mundo global postcrisis.

${ }^{17}$ El propio John Taylor (2010) ha argumentado que la política monetaria de Greenspan habría sido excesivamente relajada respecto a la regularidad empírica conocida como "regla de Taylor". En la zona euro O’Rourke y Taylor (2013) muestran cómo la estrategia de tipos del Banco Central Europeo habría encajado en los años previos a la crisis más con los bajos tipos requeri- 
contribuido a agravar "sobrecalentamiento" en esas economías, propiciando burbujas inmobiliarias y/o bursátiles. Estas distorsiones han constituído uno de los principales efectos negativos asociados a los desequilibrios externos.

Desde el inicio de la crisis, los análisis en términos de los equilibrios ahorroinversión a escala global han generado variantes con mensajes contrapuestos. Por una parte, los argumentos que plantean una reversión de la abundancia del ahorro, apuntan a una menor disposición a prestar de los superavitarios, en parte por mayores prioridades domésticas (China), combinada con mayores demandas de inversión, tanto en las propias economías emergentes como especialmente de los sectores públicos de las economías avanzadas que, según el enfoque denominado Bretton Woods III -Bibow (2010)- relevarían parcialmente al sector privado como demandantes de flujos financieros. El corolario sería el temor a un ascenso de los tipos de interés a largo plazo ${ }^{18}$, poniendo a prueba la capacidad de las políticas monetarias expansivas para mantener los tipos a corto bajos por largo tiempo.

Por otro lado, los análisis acerca de un estancamiento planteados por Summers (2013) tiene uno de sus ingredientes en una situación en la que el teórico equilibrio ahorro-inversión podría estar dándose sólo a tipos de interés negativos. Este escenario del estilo de "trampa de liquidez global" ya fue planteado por Blanchard y Milesi-Ferretti (2011) como un argumento en favor de reducir los desequilibrios globales -especialmente en la parte de responsabilidad de los países superavitarios con ahorro excesivo y/o inversión insuficiente-. Más recientemente Pisani-Ferry (2014) ha insistido en la necesidad de rebalancing aumentando la capacidad y disposición de consumo de las "nuevas clases medias globales" en las economías emergentes especialmente las superavitarias, tomando el relevo al respecto a los "consumidores de última instancia" de las economías avanzadas -si se quería evitar el escenario de estancamiento à la Summers-.

\subsection{Heterogeneidad en los flujos financieros brutos}

Diagnosticar el posicionamiento de los diferentes países tiene un ingrediente esencial en el saldo neto entre ahorro e inversión domésticos y la subsiguiente capacidad/necesidad de financiación. Pero una radiografía más precisa requiere entrar con mayor detalle en la enorme cuantía de "flujos financieros brutos" que integran las relaciones financieras internacionales. Sobre todo a la vista de la heterogeneidad de la composición de estos flujos brutos y a las diferencias al respecto entre países. Obstfeld (2012) ha insistido en la relevancia tanto de los

dos por los problemas de Alemania que los más elevados que hubiesen sido adecuados a la fase alcista en el Sur.

${ }^{18}$ El enfoque explícitamente denominado "global savings drain" por Broda et al.(2009) fue una temprana argumentación en esta línea. 
flujos brutos como de los saldos netos para tener una visión cabal tanto de las dinámicas financieras globales como de las fragilidades y problemas genera$\operatorname{dos}{ }^{19}$.

Así las implicaciones de los flujos en forma de Inversión Directa Extranjera serían -jurídica y económicamente- diferentes de las de transacciones en instrumentos de deuda, privada o pública, o de otros instrumentos financieros (créditos, derivados, etc.). El grado de compromiso inversor estable o el grado de volatilidad difieren entre fórmulas, introduciendo matizaciones que, especialmente en momentos de tensiones, resultan cruciales. Otro aspecto relacionado son los diferenciales de rentabilidades. Varios estudios detectan una sistemática mayor rentabilidad de las inversiones de Estados Unidos en el extranjero en comparación con las que entran en ese país, con explicaciones diversas que incluyen consideraciones como las del "dark matter" ya comentadas. Otra especificidad a tener en cuenta es el papel especial, ya mencionado, de los activos que se perciben como de mayor seguridad. A lo largo del tiempo ha sido un rasgo duradero el contraste entre la escasez de activos seguros frente al crecimiento más rápido de su demanda. En épocas de auge ello queda difuminado pero reaparece con fuerza en las contracciones, con efectos como el incremento de los diferenciales (primas de riesgo), tensiones en los mercados y una menor efectividad de la política monetaria convencional ya que los bajos tipos "oficiales" en parte se ven contrarrestados por crecientes spreads ${ }^{20}$.

Una moraleja importante, en la línea de "back to basics", es que el ahorro doméstico sigue siendo importante, en contraposición a lo que algunos enfoques pretendieron argumentar en los momentos de euforia precrisis acerca de que se habría alcanzado un grado de fluida canalización a escala global del ahorro hacia la inversión que convertía en crecientemente irrelevante para un país la tasa de ahorro interna ${ }^{21}$.

\subsection{Geopolítica de los desequilibrios}

En la ya clásica formulación de Bernanke (2005) la forma de ilustrar la nueva dimensión de los desequilibrios globales utilizaba la contraposición entre unas economías industrializadas que habrían pasado entre 1996 y 2003 de un superávit agregado por cuenta corriente de 46 mil millones de dólares a un déficit de 342 mil millones, mientras que las economías en desarrollo pasaban, en el mismo período, de un déficit de 87 a un superávit de 205 mil millones de dóla-

${ }^{19}$ Casos como Irlanda e Islandia fueron paradigmáticos de países con muy elevados flujos brutos en entrada y salida.

${ }^{20}$ Caballero y Emmanuel (2014) examinan las similitudes y diferencias entre una trampa de liquidez y la que denomina "safety trap" asociada al problema descrito.

${ }^{21}$ Porter et al. (2008) planteaban incluso excluir la tasa de ahorro doméstico del listado de indicadores de competitividad en base a ese argumento. 
res. En 2006, con datos del FMI, el conjunto de economías avanzadas tendrían un saldo por cuenta corriente del - 1,1\% de su PIB mientras que el conjunto de economías emergentes y en desarrollo alcanzaría una posición superavitaria del $4,8 \%$ de su PIB agregado.

Esta situación ha propiciado los principales ingredientes para interpretaciones geoestratégicas de los desequilibrios globales. Las posiciones destacadas de Estados Unidos y China al frente de los respectivos rankings de "importadores netos" y "exportadores netos" de capitales hacían inevitable las conexiones entre las situaciones financieras y las pugnas por el liderazgo mundial. Planteamientos como el de Coggan (2012) recuerdan cómo a lo largo de la historia a menudo los relevos en el poder han estado asociados -dentro de los países y a escala internacional- a los problemas de creciente endeudamiento de los "antiguos líderes" que han ido viendo como los actores emergentes se convertían en los nuevos acreedores antes de acabar relevándolos en posiciones de hegemonía. Los planteamientos iniciales que se referían a cómo "los pobres financiaban a los ricos" -con evidencias de que la renta per capita promedio de los países exportadores de capitales se situaba por debajo de la de los importadores- daba paso a interpretaciones de que eran los "nuevos ricos" los que financiaban a los "antiguos ricos...en declive", llevando la "paradoja de Lucas" más allá de su formulación inicial ${ }^{22}$. Incluso en enfoques, como el de Bretton Woods II ${ }^{23}$, que resaltan las complementariedades de intereses entre China y Estados Unidos, se apunta el debate acerca de cuándo esta conjunción táctica acabará cediendo a la contraposición estratégica de la pugna por la hegemonía. Una formulación con algunas similitudes es la de "codependencia" -en la expresión de Roach (2014)que plantea el debate acerca de si el mundo, y especialmente Estados Unidos, estaría preparado para un reequilibrio en el modelo de crecimiento de China, con la implicación de menor ahorro "excedente" disponible para ser exportado. Los debates acerca de los componentes neomercantilistas en diversos aspectos del comportamiento de los países acreedores, especialmente en China, respecto a la acumulación de reservas, tendrían asimismo lecturas en términos geopolíticos.

\subsection{Conexión con los excesos financieros}

Aunque en ocasiones se trata de contraponer el papel de los desequilibrios globales al de los "excesos" en el sector financiero como factores causa-

\footnotetext{
${ }^{22}$ Sobre la paradoja formulada por Robert Lucas en 1990 respecto a la observación de que los flujos de financiación no iban en el sentido predicho por el modelo neoclásico de crecimiento, desde los países abundantes en capital hacia los países con escasez de capital (y previsible mayor rentabilidad), que se habría visto incluso desbordada por flujos en el sentido opuesto a esa predicción, vid. los análisis sistematizados en Gourinchas y Rey (2013).

${ }^{23}$ Dooley et al. (2009)
} 
les/explicativos de la crisis financiera, las conexiones entre ambos aspectos son muy relevantes.

La tipología de esos excesos puede resumirse en el binomio que ya en 1933 Irving Fisher identificó como los rasgos asociados a las crisis financieras más importantes, incluida la Gran Depresión: por un lado, el sobreendeudamiento de unos, contrapartida de la excesiva expansión del crédito por otros, que se vería sustancialmente agravado en un escenario de deflación, en el precio de los activos y eventualmente de los bienes y servicios.

Entre los mecanismos de conexión entre desequilibrios y excesos en el sistema financiero un papel central lo ha desempeñado el bajo nivel de tipos ya señalado por Bernanke. En particular, tipos de interés sustancialmente más bajos que los que derivarían de las condiciones financieras estrictamente internas (ahorro escaso que requeriría una elevación de tipos de interés domésticos, que se vio "anestesiada" por la llegada de financiación exterior abundante y barata) El propio Fisher (1933) nos señala que easy money is the great cause of overborrowing, iniciando una secuencia de sorprendente actualidad que va desde expectativas de rentabilidades reales a otras de enriquecimiento en base a ganancias de capital (revalorizaciones), que acaban dando paso a "promociones insensatas" y casos de fraude que abusan de la credulidad de un público acostumbrado a "grandes expectativas" ${ }^{2}$. El "apetito por el riesgo" asociado a la insatisfacción de bastantes inversores ante los bajos rendimientos de los instrumentos financieros más clásicos habría impulsado a la búsqueda de opciones que ofrecían promesas de mayores rendimientos, generando mecanismos psicológicos de infravaloración de unos riesgos que, por otra parte, se trataban de mantener ocultos o al menos sustancialmente opacos. Cuando se descubría finalmente que algunos activos presentados como de la más alta calidad eran realmente "activos tóxicos" era demasiado tarde.

\subsection{Inadecuada asignación de recursos}

El argumento clásico en favor de incurrir en déficits exteriores es que si los recursos así obtenidos se destinan a financiar inversión productiva acaban generando, con creces, los ingresos suficientes para devolver lo prestado. Un razonamiento análogo trata de justificar el interés de los países superavitarios en canalizar sus excedentes a inversiones sólidamente rentables en el exterior. Por ello un aspecto crítico de los desequilibrios externos fue la creciente constatación de que en los países deficitarios los recursos que llegaron, de forma abundante y barata, del exterior se destinan a utilizaciones de productividad "subóptima". Entre las economías emergentes y en desarrollo se habla del allo-

${ }^{24}$ Los enfoques austríacos del ciclo coinciden en el papel central de tipos de interés artificialmente bajos como mecanismo central a las crisis financieras. 
cation puzzle para referirse a la correlación negativa entre llegada de fondos y productividad en su utilización. Y en varias de las economías avanzadas es asimismo conocida las conexiones entre entrada de recursos y su canalización en una cuantía notable hacía sectores como el inmobiliario y de la construcción, con indicadores de cualificación, innovación y productividad en general por debajo de la media.

Una distorsión que merece especial atención es la que se produjo en los incentivos en la asignación de recursos, que se vieron sesgados notablemente hacia los sectores non-traded que ofrecían expectativas de rentabilidades a corto plazo superiores a las que se podían esperar en sectores traded sujetos a exigencias de competitividad global que disciplinaba los precios de una forma que no existía en los non-traded. Revertir esta dinámica constituye un punto esencial en las recomendaciones de re-equilibrio global, con unas exigencias en materias de mejora de la productividad y la competitividad en las especialmente en las economías que habían llegado a posiciones deficitarias de notable importancia, desde Estados Unidos hasta, de forma destacada, las del Sur de Europa.

Pero asimismo se apuntan deficiencias que requieren correcciones en la asignación de recursos en los países superavitarios. Especialmente en el caso de China se apunta, entre otros, a los problemas del sistema financiero y al lastre que supondrían algunas de las empresas de titularidad pública cuyos compromisos de financiación habrían debilitado su eficiencia. Pettis (2013) se ha referido a la necesidad de "evitar la caída", afrontando esas importantes distorsiones.

Una implicación importante es la constatación de que en la canalización del ahorro hacia la inversión productiva desempeña un papel crítico la calidad institucional, que afecta a la eficiencia con que el sistema financiero -a escala nacional y global- realiza esa tarea esencial o, por el contrario, conlleva deficiencias y distorsiones que han agravado los desequilibrios, los excesos y el camino hacia la crisis. Los debates al respecto incluyen desde una cierta degradación en esa calidad institucional en las economías avanzadas, los intentos de recuperarla (entre otras vías mediante regulaciones que respondan realmente a los intereses generales), y los retos de las economías emergentes de contar con unas instituciones capaces de absorber y distribuir su dinámica de crecimiento ${ }^{25}$. Esta consideración enlaza con la calidad institucional de la gobernanza a escala de global que se trata en la sección siguiente.

\section{EL PAPEL DE LA COOPERACIÓN INTERNACIONAL DE POLÍTICAS: EL MARCO DEL G20}

Las apelaciones a la necesidad e importancia de la cooperación internacional

${ }^{25}$ Los problemas en China y otros emergentes, como los denominados por Roubini (2014) los "fragile Five" son aspectos mediáticos de las deficiencias a superar. 
han ido parejas a la constatación de sus dificultades de su efectiva implementación ${ }^{26}$. Los fracasos al respecto en las décadas de 1920 y 1930 (como la fallida conferencia de Londres de 1933) acentuaron las graves dificultades de la época. Tras el abandono de los tipos de cambios fijos por parte de las principales economías avanzadas en 1973 reaparecen iniciativas, especialmente a la vista de que la flexibilidad de los tipos de cambio no tenía ni el automatismo ni la gradualidad que algunas formulaciones teóricas preveían. Los debates de las décadas 1970-1980 se centran, dado ese contexto, en la coordinación en materia de tipos de cambio -como elemento central de transmisión internacional- que tiene un papel central en los "Acuerdos Plaza" de 1985, por parte del G5, para propiciar un soft landing del dólar (en vez del temido disruptivo hard landing). Analíticamente, Hamada y Oudiz- Sachs aplican las herramientas de interdependencias estratégicas para evaluar los potenciales beneficios -y dificultadesde la coordinación ${ }^{27}$. Los valores limitados obtenidos por estos últimos autores acerca de las ganancias de la coordinación (entre el 0,5 y el 1\% del PIB para Estados Unidos) no ayudaba a superar los recelos, junto a otros análisis que señalaban los problemas que podía suponer la asimetría de las perturbaciones a afrontar, así como las incertidumbres acerca del funcionamiento de la economía o las divergencias al respecto entre (eventuales) $\operatorname{socios}^{28}$ que podía dar lugar a discrepancias en los resultados previsibles de la cooperación. Que en algunos enfoques pudiese llegar a conducir a resultados contraproducentes ${ }^{29}$ era un toque tanto de atención como de escepticismo. El binomio necesidad/dificultad se conjuga de todas las formas posibles, y reaparece con fuerza tras el estallido de la crisis en 2008 con una agenda más amplia, en la que los desequilibrios externos asumen desde el inicio un papel central, como se detalla más adelante.

La excepcional gravedad de la situación condujo a la reunión en Washington del G20, en noviembre de 2008, que dejó claro desde el primer punto de su Declaración Final, la "determinación para reforzar nuestra cooperación", al tiempo que incluía entre las principales razones de haber llegado a esa situación-límite a las insuficiencias e inconsistencias en la coordinación de las políti-

${ }^{26}$ Eichengreen (2011) resume las experiencias históricas de coordinación internacional y discute las razones de sus éxitos o fracasos.

${ }^{27}$ Alonso (2010) es un claro y sistemático análisis de los debates sobre coordinación desde la década de los 1970 y sus conexiones con los problemas recientes.

${ }^{28}$ Una variante actual sería las heterogéneas posiciones de algunos bancos centrales respecto a sus estrategias monetarias y las concepciones que las subyacen. Algo similar puede argumentase acerca de los debates sobre la magnitud de los "multiplicadores fiscales". Respecto a la necesidad de construir un nuevo consenso sobre el papel de los bancos centrales, vid., entre otros, Committee on International Economic Policy and Reform (2011). Sobre las implicaciones de estas eventuales divergencias sobre las argumentaciones en favor de la coordinación, vid. Taylor (2013)

${ }^{29}$ Desde su título, un influyente artículo de Rogoff (1985) plantea esa posibilidad. 
cas macroeconómicas ${ }^{30}$. Entre los argumentos clásicos ahora aplicables en favor de buscar soluciones cooperativas cabía incluir desde afrontar externalidades negativas -del tipo de contagios financieros o transmisión internacional de ajustes contractivos- hasta los riesgos de respuestas que la Historia había demostrado peligrosas y contraproducentes -tentaciones proteccionistas, políticas cambiarias de "empobrecer al vecino"- o competencia a la baja en regulaciones financieras. Algunos de estos problemas no han dejado de estar presentes, a veces con formulaciones cambiantes, desde 2008. Así, a principios de 2014 eran objeto de controversia las "externalidades negativas" asociadas a la eventual retirada de los estímulos monetarios en Estados Unidos, así como los spillover negativos asociados a la ralentización de las economías emergentes, mientras que, en positivo, las primeras formulaciones de lo que se esperaba fuese un ambicioso plan de acción Brisbane resaltaban cómo las medidas propuestas tendrían efectos internos positivos en los países implicados pero asimismo se insistía en que "la actuación conjunta podría producir spillovers de crecimiento beneficiosos a medio y largo plazo" $"$.

\subsection{Corregir los desequilibrios externos}

La corrección de los desequilibrios externos ha sido un punto central de una agenda del G20 que se ha ido ampliando con el tiempo. Desde la cumbre de Pittsburgh (2009) se asume oficialmente un "Marco para un crecimiento sólido, sostenible y equilibrado" ${ }^{32}$ que se complementa con un mecanismo de Mutual Assessment Process (MAP) encargando al FMI la presentación de periódicos análisis e informes acerca de los desarrollos y políticas en los países del G20 ${ }^{33}$, a efectos de poder ejercer una "supervisión multilateral" acerca de los compromisos asumidos en las reuniones del G20 y en particular para el seguimiento de los desequilibrios.

En su formulación inicial más básica, posteriormente detallada y sofisticada, pero esencialmente vigente, se centra en dos familias de recomendaciones, una dirigida a países con déficit por cuenta corriente y otra para países con superávit exterior. A los países deficitarios se les recomienda, por un lado, avanzar hacia una menor dependencia de un ahorro exterior que la historia demuestra que,

${ }^{30}$ Puntos 1 y 4 de la Declaración final de la cumbre del G20, Washington, noviembre de 2008, disponible en: http://www.g20.utoronto.ca/2008/2008declaration1115.html

${ }^{31}$ FMI (2014), que cifra esos spillover positivos (especialmente en difusión tecnológica y mejoras de productividad) en una tercera parte de las ganancias esperadas del Plan de Acción para el conjunto de la economía mundial.

${ }^{32}$ Sobre cronologías y sistemáticas básicas de la coordinación internacional en el marco del G20, vid. Estrada (2012) y Pisani-Ferry (2012).

${ }^{33}$ Los documentos del G20 pueden verse en: http://www.g20.org/ y los documentos preparados por el FMI para el G20 pueden verse en: http://www.imf.org/external/np/exr/facts/g20map.htm 
más allá de ciertos umbrales, no puede darse por garantizado que va a ser abundante y barato, como la crisis ha evidenciado con crudeza para bastantes países. Ello requiere adecuar el gasto, tanto privado como público, a las nuevas realidades y asimismo pautas de desendeudamiento que, en el sector público, se traducen en la denominada consolidación fiscal. Y por otra parte, enlazando con el epígrafe anterior, reciben la recomendación de incentivar la asignación de recursos hacia los sectores y actividades más orientados a la competencia internacional (traded) en detrimento de los sectores menos sujetos a competencia exterior (non traded) que fueron los protagonistas del auge con pies de barro que condujo a la crisis. Las recomendaciones para los países con superávit externo van en las líneas opuestas: más orientación en la asignación de recursos hacia sectores non traded, una estrategia más orientada al gasto (consumo, en el caso de China, y asimismo inversión en el caso de Alemania); en los países emergentes y en desarrollo más avances en programas de protección social y, asimismo con referencia a China, criterios de mercado en la determinación del tipo de cambio.

Los logros al respecto han sido valorados de forma positiva pero modesta. Así, el comunicado del G20 de febrero de 2014 se refería a cómo la economía global seguía estando "lejos de alcanzar un crecimiento fuerte, sostenible y equilibrado", con mención expresa de los "continuing global imbalances", para enmarcar las propuestas del que deberá ser el Plan de Actuaciones de Brisbane con la finalidad de estimular el crecimiento mundial de forma coordinada y con reducción de los desequilibrios. Con más detalle, el documento del FMI (2014) afirma que la reducción de los desequilibrios externos ha sido apreciable desde el inicio de la crisis, reflejando en parte ajustes "saludables" como un rebote en el ahorro privado y, más recientemente, mejoras en los saldos fiscales de economías con déficit exterior, así como demanda interna más resiliente en algunas economías emergentes clave; pero asimismo una parte considerable de la reducción de los desequilibrios refleja también una "demanda más débil en economías avanzadas con déficit, al tiempo que los ajustes hacia políticas deseables a medio plazo han sido modestos en general y han desempeñado solo un pequeño papel hasta ahora en la reducción de los desequilibrios globales".

\section{2. ¿Rendimientos decrecientes a la cooperación?}

Tras el momento álgido en el clima de cooperación internacional que propiciaron las urgencias de la vertiginosa situación a finales de 2008 y en 2009, las asimetrías en la magnitud y velocidad de la recuperación debilitaron la efectividad del G20, haciendo aplicables expresiones como la de "rendimientos decrecientes en la cooperación internacional". ¿Qué sucedió?

Desde el FMI, Ostry y Ghosh (2013) han sistematizado cómo los momentos de urgencias compartidas son propicios a comportamientos cooperativos del estilo de "arrimar todos el hombro", que se relajan cuando la presión de las ur- 
gencias -y el desconcierto e menudo asociado- se va disipando. En especial destacan cómo la coordinación es más fácil cuando implica que todos los participantes actúen en el mismo sentido (medidas expansivas en los momentos iniciales de la crisis) pero aumentan las dificultades para mantener la coordinación cuando implica hacer cosas diferentes, en la línea de recomendaciones divergentes a países superavitarios y deficitarios. En una línea similar ese análisis valora los esfuerzos para evitar tentaciones proteccionistas, pero asimismo constata las serias dificultades para avanzar en la línea de cerrar la ronda Doha (un objetivo formalmente explicitado como deseable a corto plazo ya en la declaración del G20 de noviembre de 2008) ${ }^{34}$. Asimismo las controversias periódicas sobre alineamientos correctos de los tipos de cambio revelarían deficiencias en la operatividad de los mecanismos de cooperación.

Adicionalmente, con una recuperación modesta y heterogénea (a varias velocidades) reaparecen las prioridades nacionales y se debilitan los incentivos a la cooperación. Los documentos del MAP clasifican oficialmente a los países del G20 en 5 categorías atendiendo a su nivel de desarrollo y a su posición exterior superavitaria o deficitaria (más una quinta categoría de exportadores de petróleo y otras materias primas). El hecho de que actores de la envergadura de China, Estados Unidos, Alemania e India encabecen los listados de los grupos resultantes de cruzar los dos primeros criterios muestra la heterogeneidad de intereses y la asimetría de posiciones.

Una tercera fuente de problemas deriva de la asimetría en la presión para corregir los desequilibrios externos y sus eventuales impactos contractivos. El punto de partida es que una corrección en principio simétrica de los superávits de unos y de los déficits exteriores de otro requiere ajustes en sentidos opuestos, en las líneas resumidas en el epígrafe anterior. Y ello se enfrenta a la asimetría entre la capacidad para inducir a los países deficitarios y a los superavitarios a emprender sus respectivos ajustes. A los países deficitarios, con necesidad de financiación exterior, se les puede presionar, convencer o inducir a realizar los ajustes contractivos (reducción de la absorción) por la vía de la condicionalidad $\mathrm{y} / \mathrm{o}$ costes de acceder a la financiación que siguen requiriendo. Por el contrario, a los países superavitarios, con "capacidad de financiación", es mucho más difícil encontrar mecanismos análogamente operativos de inducción a los ajustes (más expansión) si ello no encaja con sus prioridades internas (o con un inusual "sentido de responsabilidad global"). Un resultado de esa asimetría es que si finalmente los países deficitarios efectivamente ajustan a la baja, mientras que los países superavitarios, que deberían hacerlo al alza, se resisten, el efecto neto sobre el sistema sería contractivo, y, eventualmente, podría implicar ries-

\footnotetext{
${ }^{34}$ En el ámbito comercial los sucesivos informes de Global Trade Alert (decimocuarta versión en septiembre de 2013, en www.globaltradealert.org ) dan una imagen menos optimista acerca de las dinámicas proteccionistas.
} 
gos deflacionistas ${ }^{35}$. Este es el resultado explicitado por UNCTAD (2013) y, a escala europea, el temor expresado en estudios y documentos de la Comisión Europea $(2012,2014)$ respecto a la continuidad de algunos importantes superávits externos. Otra asimetría, entre los países deficitarios, sería la que estaría permitiendo al país cuya moneda funciona como de reserva internacional eludir con más facilidad los ajustes, de modo que, en conjunto, la carga de corregir los desequilibrios recaiga de forma desproporciona sobre los deficitarios sin moneda de reserva.

\section{3. ¿Un nuevo impulso a la cooperación internacional?}

En febrero de 2014 los resultados de la reunión del G20 a nivel de Ministros de Finanzas y Gobernadores de Bancos Centrales comienzan a concretar, en base a documentos preparados principalmente por el FMI y la OECD, la estrategia anunciada como plan de acción de Brisbane. Se trata de un amplio conjunto de medidas, combinando las líneas de mejorar el output potencial y de acercar el nivel efectivo de actividad a ese output, reduciendo las brechas al respecto. Se trata de afrontar los problemas de fondo, propiciando mejoras de eficiencia, propiciando incentivos y estímulos, y recuperando niveles de compromisos conjuntos en favor de un crecimiento no sólo mayor sino más sólido, constituyendo la reducción de los desequilibrios inapropiados uno de los efectos y objetivos.

¿Se trata de una nueva retórica o hay razones para un mayor optimismo? En comparación con los argumentos anteriores podría alegarse que la ralentización en algunas economías emergentes hace algo más simétrica la dinámica de una recuperación que se percibe como frágil y que los sesgos recesivos pueden estar teniendo una creciente constatación. Ello constituiría un escenario más propicio para avanzar en la cooperación internacional, pero subsisten tensiones que obligan a la mayor cautela.

En todo caso, los aspectos relacionados con los desequilibrios externos tienen un papel relevante en los intentos de revitalizar la coordinación internacional. Así, se reitera (FMI, 2014) la necesidad de "evitar un resurgimiento de los desequilibrios globales a medida que avance la recuperación...", al tiempo que se ratifican los compromisos con las políticas dirigidas a estimular la demanda

${ }^{35}$ Como han recordado, en conexión con los debates actuales, Skidelsky-Joshi (2010), la asimetría entre unos ajustes que eran de facto "obligatorios para el deudor, voluntarios para el acreedor" fue un tema recurrente en los análisis de Keynes acerca de los problemas de las décadas entre las dos guerras mundiales. El Plan Keynes para una Clearing Union de 1941, base de las posiciones que iba a defender en la conferencia de Bretton Woods, trataba de establecer incentivos para inducir también a los países superavitarios a su parte alícuota del ajuste evitando que éste recayese exclusivamente en los deudores. 
interna en las economías superavitarias y a desplazarla hacia la demanda externa en las deficitarias". E incluso reconociendo que las políticas de rebalancing contribuyen "sólo" al crecimiento a medio plazo vía el estímulo de la inversión en las avanzadas con superávit, se argumenta que son necesarias para "reducir los riesgos para la sostenibilidad del crecimiento que provienen de desequilibrios internos y externos, incluyendo los riesgos de crisis financieras". Un enfoque no muy alejado del que sostenía UNCTAD (2013) al contraponer los riesgos de continuar con el baseline (ajustes asimétricos con sesgo recesivo) con un escenario de políticas expansivas (más pronunciadas en los países superavitarios), con reducción notable de los desequilibrios externos.

$\mathrm{Y}$ a la hora de valorar el papel de la coordinación internacional, se insiste en que "la actuación conjunta podría producir spillovers de crecimiento beneficiosos a medio y largo plazo"... así como "incluso más importante, la actuación conjunta puede también reducir los riesgos de renovadas turbulencias globales tanto reduciendo los desequilibrios externos como las distorsiones internas y reforzando la confianza de los mercados" (FMI, 2014). Y se concluye con el mensaje de que el "compromiso demostrado con estas actuaciones conjuntas enviaría una fuerte señal a los participantes en el mercado acerca de la importancia de los policymakers globales conceden al objetivo de reducir los riesgos globales a través de la cooperación de políticas"

\section{CONSIDERACIONES FINALES}

El retorno a un crecimiento (más) equilibrado es el escenario oficialmente asumido como deseable. Hay buenas razones para ello en términos de un mejor funcionamiento de la economía global que reduzca los riesgos de crisis financieras y haga más perdurable el crecimiento. Los escenarios alternativos son menos deseables, desde los peligros asociados a varias formas de desglobalización (desde riesgos proteccionistas en el ámbito comercial o, eventualmente, en el financiero, hasta fricciones geopolíticas), a las tentaciones de retorno a un business as usual que trata de aprovechar la recuperación para, simplemente, (volver a) olvidar las lecciones de la crisis, manteniéndose el germen de los desequilibrios y fragilidades con el riesgo de volver a poner en marcha una dinámica hacia una subsiguiente crisis. Las declaraciones del G20, especialmente en 2008-2009 y más recientemente en 2014, van en la dirección correcta, siendo el principal problema la percepción de que, más allá de momentos especialmente críticos, su aplicación es parcial, limitada y en ocasiones con resultados asimétricos y recesivos que dificultan los incentivos a avanzar en la cooperación internacional.

Los enfoques más recientes de los desequilibrios externos van más allá de su papel como síntoma -y más allá de un cierto umbral, también como causa- de fragilidades, para profundizar en los factores que les subyacen y enlazar con las 
fuentes del crecimiento y la competitividad. Evaluar los desequilibrios conduce no ya a una simple radiografía sino a un cada vez más completo scanner de la economía global que debería facilitar la cooperación internacional, pese a las dificultades económicas y geopolíticas para ello. En última instancia se trata de un problema de gobernanza en un mundo más multipolar en que los comportamientos cooperativos tienen más potencial de aportaciones positivas pero al mismo tiempo más dificultades políticas de implementación. Avanzar adecuadamente en la resolución de los problemas de los desequilibrios -de las causas profundas y no (sólo) de los síntomas- es una de las vías para comprobar que el G20, con el apoyo de los organismos multilaterales y regionales, es un instrumento eficiente para afrontar la gobernanza global y eludir los temores de continuar en unos peligrosos rendimientos decrecientes.

\section{REFERENCIAS BIBLIOGRÁFICAS}

ALONSO, JOSÉ ANTONIO (2010). "Coordinación macroeconómica y desequilibrios globales", Pensamiento Iberoamericano, n.6, 2ª época, 2010/1 (p. 285-314)

BERNANKE, BEN (2005). "The Global Saving Glut and the U.S. Current Account Deficit", Homer Jones Lecture, abril de 2005, http://www.federalreserve.gov/ boarddocs/speeches/2005/200503102/ [Último acceso, 20 de marzo de 2014]

BIBOW, JÖRG (2010). "Bretton Woods 2 is Dead, Long Live Bretton Woods 3?", Levy Economics Institute working paper 597, mayo de 2010.

BLANCHARD, OLIVIER y MILESI-FERRETTI, GIAN MARIA (2009). "Global Imbalances: In Midstream?", IMF Staff Position Note 09/29, diciembre 2009.

BLANCHARD, OLIVIER y MILESI-FERRETTI, GIAN MARIA (2011). "(Why) Should Current Account Balances Be Reduced?", IMF Staff Discussion Note, SDN/11/03, marzo de 2011.

BRODA, CHRISTIAN, GHEZZI, PIERO y LEVY-YEYATI, EDUARDO (2009). "The new global balance", Barclays Capital, Economics Research, 23 marzo de 2009.

CABALLERO, RICARDO (2010). "The Other "Imbalance" and the Financial Crisis", NBER working paper 15636, enero 2010.

CABALLERO, RICARDO y FARHI, EMMANUEL (2014). “The Safety Trap”, NBER working paper 19927, febrero 2014.

COGGAN, PHILIP (2012). Paper Promises: Debt, Money and the New World Order, Public Affairs and Penguin Group, 2012.

COMISIÓN EUROPEA (2012). Current Account surpluses in the EU, European Economy, n. 9, 2012.

COMISIÓN EUROPEA (2014). "Results of in-depth reviews on the prevention and correction of macroeconomic imbalances", 5 de marzo de 2014, http://ec.europa.eu/ economy_finance/economic_governance/macroeconomic_imbalance_procedure/ind ex_en.htm [Último acceso, 20 de marzo de 2014].

COMMITTEE ON INTERNATIONAL ECONOMIC POLICY AND REFORM (2011). Rethinking Central Banking, Brookings Institution, septiembre de 2011, disponible en: 
http://www.brookings.edu/research/reports/2011/09/ciepr-central-banking. [Último acceso: 20 de marzo de 2014].

DOOLEY et al. (2009). "Bretton Woods II Still Defines the International Monetary System", NBER working paper 14731, febrero 2009.

EICHENGREEN, BARRY (2011). "International Policy Coordination: the Long View", NBER working paper 17665, diciembre de 2011.

EICHENGREEN, BARRY (2014). " A Requiem for Global Imbalances”, Project Syndicate, 13 de enero de 2014, http://www.project-syndicate.org/commentary/barryeichengreen-notes-that-a-decade-after-external-imbalances-emerged-as-a-

supposed-threat-to-the-global-economy-the-problem-has-disappeared [Último acceso, 20 de marzo de 2014].

ESTRADA, ÁNGEL (2012). "Nuevas herramientas para la gobernanza económica global: marco para el crecimiento y supervisión de desequilibrios", Boletín Económico, Banco de España, septiembre de 2012 p. 65-79).

EUROPEAN CENTRAL BANK (2014). "To What Extent Has the Current Account Adjustment in the Stressed Euro Area Countries Been Cyclical or Structural?", en Boletín Mensual, enero de 2014, Box 5, p. 47-50.

FISHER, IRVING (1933). "The Debt-Deflation Theory of Great Depressions", Econometrica, vol. 1, issue 4, octubre 1933 (p. 337-357).

FMI (2013 a). "2013 Pilot External Sector Report”, FMI, junio de 2013.

FMI (2013b). Update on Global Prospects and Policy Challenges, G20, septiembre de 2013, disponible en: http://www.imf.org/external/np/g20/pdf/2013/090513.pdf [Último acceso, 18 de marzo de 2014].

FMI (2013 c). "Imbalances and Growth", documento preparado para el G20, septiembre de 2013, disponible en: http://www.imf.org/external/np/g20/map2013.htm [Último acceso, 20 de marzo de 2014].

FMI (2014). "Global Prospects and Policy Challenges", preparado para el encuentro de Ministros de Finanzas y Gobernadores de Bancos Centrales del G20 de Sydney, 2223 de febrero de 2014, disponible en: http://www.imf.org/external/np/g20/pdf/ 2014/021914.pdf [Último acceso, 20 de marzo de 2014].

G20 (2009). G20 Leaders' Statement: The Pittsburg Summit, septiembre de 2009, http://www.g20.utoronto.ca/2009/2009communique0925.html [Último acceso 19 de marzo de 2014].

G20 (2013). G20 Leaders' Declaration, Saint Petersburg Summit, septiembre de 2013, http://www.g20.utoronto.ca/2013/2013-0906-declaration.html [Último acceso 19 de marzo de 2014].

G20 (2014). Communiqué - Meeting of Finance Ministers and Central bank Governors, Sydney 22-23 febrero de 2014, disponible en: www.g20.org [Último acceso, 20 de marzo de 2014].

GOURINCHAS, PIERRE-OLIVIER y REY, HELÈNE (2013). External Adjustment, Global Imbalances and Valuation effects, NBER working paper 19240, junio 2013

HAUSMANN, RICARDO y STURZENEGGER, FEDERICO (2007). "The missing dark matter in the wealth of nations and its implications for global imbalances", Economic Policy, vol. 22, n. 51, julio 2007 (p. 469-518).

OBSTFELD, MAURICE (2012). Does the Current Account Still Matter?, American Economic Review, vo. 102, n. 3, may 2012 (p. 1-23). 
OLLIVAUD, PATRICE y SCHWELLNUS, CYRILLE (2013). The Post-Crisis Narrowing of International Imbalances - Cyclical or Durable?, Economics Department Working Papers n. 1062, junio 2013.

O'ROURKE, KEVIN y TAYLOR, ALAN (2013). Cross of Euros, Journal of Economic Perspectives, vol. 27, n. 3, summer 2013, pp. 167-192.

OSTRY, JONATHAN y GHOSH, ATISH (2013). Obstacles to Internacional Policy Coordination, and How to Overcome Them, IMF Staff Discussion Note DSN 13/11, diciembre 2013.

PETTIS, MICHAEL, (2013). The Great Rebalancing: Trade, Conflict, and Perilous Road Ahead for the World Economy, Princeton University Press, 2013.

PISANI-FERRY, JEAN (2012). "Macroeconomic Coordination: What Has the G-20 Achieved?", disponible en: http://www.bruegel.org/publications/publication-detail/publication/726-macroeconomic-coordination-what-has-the-g-20-achieved/ [Último acceso, 20 de marzo de 2014].

PISANI-FERRY, JEAN (2014). "The New Growth Conundrum", Project Syndicate, 27 de febrero de 2014, disponible en: http://www.project-syndicate.org/commentary/jeanpisani-ferry-warns-that-the-advanced-countries-may-be-headed-toward-a-future-ofhigh-tech-inequality [Último acceso, 20 de marzo de 2014].

PORTER, MICHAEL et al. (2008). "Moving to a New Global Competitiveness Index", capítulo 1.2 del Global Competitiveness Report 2008-2009, World Economic Forum.

ROACH, STEPHEN (2014). Unbalanced - The Codependency of America and China, Yale University Press, 2014.

ROGOFF, KENNETH (1985). "Can International Monetary Policy Coordination be Counterproductive?", Journal of Internacional Economics, vol. 18, 1985 (p. 199-217)

ROUBINI, NOURIEL (2014). "The Trouble with Emerging Markets", Project Syndicate, 31 de enero de 2014, http://www.project-syndicate.org/commentary/nouriel-roubiniexplains-why-many-previously-fast-growing-economies-suddenly-find-themselvesfacing-strong-headwinds [Último acceso: 19 de marzo de 2014].

SKIDELSKY, ROBERT y JOSHI, VIJAY (2010). "Keynes, Global Imbalances, and International Monetary Reform, Today", en Claessens et al. (eds), Rebalancing the Global Economy: A Primer for Policymaking", A VoxEU.org Report, 2010. Disponible en: http://www.voxeu.org/epubs/cepr-reports/rebalancing-global-economy-primer-policymaking

SUMMERS, LARRY (2013). "Why stagnation might prove to be the new normal", 15 de diciembre de 2013, http://larrysummers.com/commentary/financial-timescolumns/why-stagnation-might-prove-to-be-the-new-normal/ [Último acceso: 19 de marzo de 2014].

TAYLOR, JOHN (2010)."Getting Back on Track: Macroeconomic Policy Lessons from the Financial Crisis", Federal Reserve Bank of St. Louis Review 92(3), junio de 2010 (p. 165-176).

TAYLOR, JOHN (2013). "International monetary policy coordination: past, present and future", Bank for International Settlements, working paper n. 437, diciembre 2013.

UNCTAD (2013). Trade and Development Report ,2013: Adjusting to the changing dynamics of the world economy, UNCTAD,/TDR/2013, UNCTAD, septiembre de 2013. 
